\section{Diving into the Details: Constructing a Framework of Random Call Components}

\author{
Alex H. Waugh and Tessa C. Andrews*
}

Department of Genetics, University of Georgia, Athens, GA 30602

\begin{abstract}
Random call is a randomized approach to select a student or group of students to share their thinking with the whole class. There are potential costs and benefits of random call in undergraduate courses, yet we lack insight about how this strategy is actually implemented and why instructors choose to use it. We interviewed 12 college biology instructors who use random call in courses with $\mathbf{5 0}$ or more students. Qualitative content analysis revealed why these instructors chose to use random call, the specific ways they implemented random call, and the reasoning behind their implementation. Instructors used random call to increase the diversity of voices heard in the classroom and to hold students accountable for working. Random call users showed concern about student anxiety and took specific steps to mitigate it. We break random call down into a series of components, identify the components that our participants considered most critical, and describe the reasoning underlying random call components. This work lays a foundation for future investigations of how specific random call components influence student outcomes, in what contexts, and for which students.
\end{abstract}

\section{INTRODUCTION}

Students explaining their thinking to the class is a common feature of undergraduate active-learning classrooms. One instructional approach to select speakers is random call, which occurs when an instructor selects a student or group of students to share their thinking with the class and everyone has an equal probability of being chosen. Random call is often contrasted with asking for volunteers. Random call is also related to cold calling. There is currently no agreed-upon definition of cold calling, but it is generally used to refer to the instructor calling on a student by name (e.g., Eddy et al., 2015).

Prior research has identified multiple potential benefits and costs of using random call as an instructional strategy. Random call can increase the diversity of students heard in courses. For example, gender disparities persist in undergraduate biology education. Males are more likely to answer questions in front of the class when an instructor asks for volunteers (Eddy et al., 2014). In some courses using volunteers, the proportion of females enrolled in a course was more than twice as large as the proportion of females heard (Eddy et al., 2014). Disparities in who shares their thinking with the class disappear in courses using random call (e.g., Martin et al., 2006; Eddy et al., 2014). Additionally, random call may work against implicit gender biases students hold about who does particularly well in biology courses, at least among female students (e.g., Grunspan et al., 2016).

Random call can also increase student accountability to participate fully during class. Verbalizing one's reasoning and responding to reasoning presented by peers contributes to deep, conceptual learning (e.g., Chi and Wylie, 2014). However, this level of cognitive engagement is challenging, and thus students may need additional motivation. Random call may provide that motivation. Knight and colleagues (2016) compared two sections of a course in which students regularly discussed clicker questions in small groups and then shared their reasoning with the class. One section asked
Cynthia Bauerle, Monitoring Editor Submitted Jul 9, 2019; Revised Feb 6, 2020; Accepted Feb 20, 2020

CBE Life Sci Educ June 1, 2020 19:ar14

DOI:10.1187/cbe.19-07-0130

*Address correspondence to: Tessa C. Andrews (tandrews@uga.edu).

(c) 2020 A. H. Waugh and T. C. Andrews. CBE-Life Sciences Education @ 2020 The American Society for Cell Biology. This article is distributed by The American Society for Cell Biology under license from the author(s). It is available to the public under an Attribution-NoncommercialShare Alike 3.0 Unported Creative Commons License (http://creativecommons.org/licenses/ by-nc-sa/3.0).

"ASCB®" and "The American Society for Cell Biology ${ }^{\circledR}$ " are registered trademarks of The American Society for Cell Biology. 
for volunteer groups and the other randomly selected groups to share. Randomly selecting groups resulted in a greater proportion of small-group discussions in which students exchanged reasoning and posed questions to one another than did asking for volunteer groups (Knight et al., 2016). Students in another undergraduate biology course reported that random call increased their engagement, including their attendance, preclass preparation, and involvement in small-group discussions (Broeckelman-Post et al., 2016). These results suggest that using random call may create conditions in which students are more likely to engage in cognitive work that is important to their learning.

As mentioned earlier, there are also potential costs of random call as an instructional strategy (England et al., 2017; Cooper et al., 2018). Random call may result in high levels of anxiety among some students, and anxiety can negatively influence student outcomes in science. Interviews with students enrolled in two large college biology courses revealed that about $60 \%$ of students felt that the use of random or cold call increased their anxiety (Cooper et al., 2018). These students worried about being negatively evaluated by others. Researchers hypothesize that this worry may be so distracting that some students are unable to cognitively engage in the work being asked of them and may therefore perform more poorly (Cooper et al., 2018). Some students report that they are unable to clearly articulate their thoughts when called on because of the anxiety they experience (Cooper et al. 2018). These researchers hypothesize that random call could lead to inequitable classroom experiences if some students, such as female students, experience more anxiety than others when they are randomly selected to speak in front of the class (Cooper et al., 2018). Another study found that cold call caused students in large introductory biology courses significantly higher anxiety than did other active-learning strategies (e.g., group work, worksheets, clickers; England et al., 2017). Notably, students in this study reported just as much anxiety about volunteering answers as they did about cold calling (England et al., 2017), so the source of anxiety may be more about talking in front of peers than being selected to do so.

In some cases, random call may reduce the apprehension students feel about participating in class (Eddy et al., 2015). Comparisons of undergraduate business courses indicated that students in a course using high levels of random call became more willing to volunteer answers to instructor questions and to pose questions to the instructor in front of the class. In comparison, students in a course using low levels of random call did not change in their willingness to volunteer or pose questions over the semester (Dallimore et al., 2013). Importantly, the instructor in this course used several strategies to increase student comfort, which likely provided the students with positive exposure to random call. High levels of random call may not reduce apprehension if students' experiences are negative (Heimberg et al., 2010). Instructors studied in Dallimore et al. (2013) established that students were expected to participate in class discussions early in the course, provided students time to think and discuss with peers before being called on, and fostered an encouraging learning environment.

Clearly, the existing literature lacks consensus about how random call affects students. Currently, we cannot reconcile the results of different studies because the specific ways in which random call is being implemented are unclear. How random call is used in a course may be crucial to the costs and benefits for students (e.g., Dallimore et al., 2004b). For example, in a large introductory biology course using frequent random call (20 times per class session), some students felt anxious about being "put on the spot" when they were selected, but overall students felt more positive than negative about the use of random call in the class. Notably, the instructor in this course encouraged students to ask the instructor a question if they were selected and did not feel prepared to share a response (Broeckelman-Post et al., 2016). Additionally, students often had the option to discuss a question with their peers and raise their hands to speak in front of the class later in the class session if they felt unprepared to speak when they were first selected. This raises important questions about whether and how random call can be implemented to maximize the benefits (e.g., accountability to participate, diversity in whose voices are heard the classroom) and minimize the costs (e.g., student anxiety).

A key gap in our current knowledge is how random call is actually being implemented in college science, technology, engineering, and mathematics (STEM) courses and why instructors choose to use it. Without additional clarity regarding the specific ways in which random call is implemented, we cannot empirically weigh the benefits and costs for students, nor can we make comparisons across studies.

We are guided by work that aims to identify and describe "critical components" of research-based strategies (Stains and Vickrey, 2017; Scanlon et al., 2019). Critical components are elements of a strategy that are central and necessary to its impact. Ultimately, components are confirmed as critical in reference to their impact on student outcomes, such as learning, sense of belonging, persistence, and more. Some strategies, such as peer instruction, have been described in detail by developers (Mazur and Hilborn, 1997) and have a large body of empirical evidence that can be used to identify the components critical to the impact of the strategy (e.g., Vickrey et al., 2015). Being able to identify which components are critical to achieving the promised outcomes of an active-learning strategy is increasingly important as more instructors adopt these strategies if we wish to avoid misuse of evidence-based practices (e.g., Andrews et al., 2011; Dancy et al., 2016).

The existing literature on random call is insufficient to identify its critical components. In fact, researchers do not even use a common definition, making it impossible to compare outcomes of random call across studies. This work aims to develop a framework of the practices that constitute random call and to generate hypotheses about what components are critical to student outcomes. User interviews are a strong starting place for developing hypotheses about the critical components of a strategy (e.g., Hall and Loucks, 1978). User interviews are particularly valuable for discovering how and why a strategy is implemented in real-world contexts (e.g., Scanlon et al., 2019). We strategically sampled instructors who used random call. Our participants felt that this strategy was effective for them. This makes them uniquely qualified to provide insights into components of random call that are important to their implementation of this practice. The results of this study should not be generalized beyond the study sample and should not be taken as evidence of the impact of random call on students. Rather, we used in-depth analysis of the thinking present in a specific 
TABLE 1. Participant class size, institution type and location, and level of biology course discussed in the interview

\begin{tabular}{lcll}
\hline Pseudonym & Class size(s) & \multicolumn{1}{c}{ Institution type and location } & Level of course \\
\hline Ramona & 230 & Very high research activity, southeastern United States & Introductory \\
Rebecca & 180 and 60 & Very high research activity, western United States & Introductory and upper-division \\
Rhonda & 175 & Same institution as above & Upper-division \\
Rita & 70 & Very high research activity, southeastern United States & Introductory \\
Robin & 70 & Exclusively undergraduate, midwestern United States & Introductory \\
Renee & $100-550$ & Very high research activity, western United States & Introductory \\
Robert & $500-700$ & Same institution as above & Introductory \\
Rachel & $500-700$ & Same institution as above & Introductory \\
Rose & 100 & Very high research activity, southeastern United States & Introductory \\
Ruth & 120 & Very high research activity, southwestern United States & Upper-division \\
Regina & 330 & Very high research activity, southeastern United States & Introductory \\
Rhea & b & Same institution as above & Introductory \\
\hline
\end{tabular}

aParticipants at the same institution are listed together.

${ }^{b}$ These two participants were part of the broader study of instructor thinking.

sample of instructors to create a framework of potential components of random call and to generate hypotheses about what components of random call may be critical to student outcomes. This study lays the groundwork for future larger-scale investigations of how hypothesized critical components influence student outcomes, and in what contexts.

This study investigated how a sample of college biology instructors think about and use random call, with the goal of answering two specific research questions:

1. How do random call users weigh benefits and costs in making decisions to use random call?

2. How do these instructors implement random call in the classroom?

In addressing the second question, we focused particularly on identifying potential critical components of the implementation of random call. We also studied the reasoning behind the specific ways in which instructors implemented random call, which included how they considered student anxiety as they made instructional decisions. We focused on what participants said about their practices, rather than observing their practices, in order to understand the rationales behind their teaching decisions.

\section{METHODS \\ Participants}

We recruited two distinct samples of participants who used random call, totaling 12 instructors of undergraduate life sciences courses with 50 or more students at 8 institutions (Table 1 ). We included individuals from two different samples because the research approaches yielded similar data about their thinking and use of random call.

One sample consisted of two participants from one institution who were part of a broader study of instructor thinking. We selected these participants for the broader study, because they self-identified and were seen by colleagues as active-learning instructors and taught at schools in the southeast United States. These instructors used random call and their careful thinking about this practice inspired additional data collection.

We recruited 10 additional participants from seven institutions for a more focused study of instructor thinking about random call (Table 1). We used snowball sampling to identify college biology instructors who use random call in large courses. We began by contacting individuals who had contributed to a conversation about cold calling on a Listserv of a professional society related to undergraduate biology education. We asked these individuals to participate and to recommend other biology instructors who used random call. We explicitly defined random call as "a randomized approach to select a student or group of students to share their thinking with the whole class." All research was conducted under IRB protocols 00004989 and 00006486 at the University of Georgia.

\section{Data Collection}

We collected data via semistructured interviews. Interviews were transcribed verbatim and served as the raw data analyzed in this study. Interviews differed between the two samples of participants. The broader study asked questions about many aspects of teaching, including a few questions about the use of random call. In contrast, the more focused study asked only questions about random call and elicited more detail about participants' implementation of this practice.

The broader study of instructor thinking aimed to elicit thinking that occurs while planning, implementing, and reflecting on active-learning instruction in large college biology courses (Andrews et al., 2019). Author T.C.A. interviewed participants before and after a target class session, and participants discussed random call in both interviews. The preclass interview targeted thinking related to planning the course and the target class session. The postclass interview targeted participants' rationales for in-the-moment teaching decisions and their reflections, using video clips from the target class as stimuli (e.g., Alonzo and Kim, 2016). The pre- and postclass interviews each lasted approximately 60 minutes and covered a variety of topics related to active learning. Only a small portion of these interviews contained data related to random call, and we limited our analyses to these sections. T.C.A. also observed the target class session, providing additional insight about their use of random call.

The interviewer asked participants to explain the rationale behind using random call and to describe how they used random call. Participants discussed random call in response to this question on the preinstruction interview, "How do you typically interact with students during class?" They discussed random 
call in the postinstruction interview as they reflected on video clips that showed them using random call. The goal of the interview was to learn why participants used random call by asking questions such as "Talk to me about your rationale for using random call in your class" and "So you're using random call here. Why did you choose to do that at that time?" Another goal was to learn how they used random call by observing their use of random call in the classroom and asking questions like "How do you call on groups?" and "How often do you use random call to ask a student to explain their thinking?"

The more focused study aimed to understand how and why random call users implement this strategy. Thus, we may have more detailed data about how these participants used random call and their rationales for the specific ways in which they implemented random call. We asked participants to briefly describe how they used random call via email during participant recruitment, both to screen for participants whose instructional practices aligned with how we defined random call and to establish basic information about how the participants used random call. Author A.H.W. started each interview by asking participants for general information about how they use random call. A.H.W. asked follow-up questions to fully understand the random call implementation described by participants. Example questions include: "Do you call on individuals or groups?" and "When is the first time during the semester that you use random call?" (see full protocol in Supplemental Material). We also asked participants to explain what motivated them to use random call. Given concerns about student anxiety associated with random call, we also said, "Some instructors worry about students feeling anxious when random call is used. How do you think about that?" We ended the interviews by asking participants to respond to this question, "If you had to boil it down, what advice would you give to an instructor who wants to start using random call in a large course?" This question elicited participants' thinking about both their core reasons for using random call and components of their implementation they saw as particularly important.

\section{Data Analysis}

The goal of our data analysis was to identify and describe reasons why participants use random call, the specific components of their implementations of random call, and why they choose to use those components. From these data, we sought to understand which components our participants considered most critical.

Qualitative Content Analysis. We conducted qualitative content analysis to address our research questions. This analysis was highly collaborative and iterative. The first phase of our analysis consisted of reading each interview transcript and identifying sections of each transcript relevant to our study. Transcripts from the focused study did not require this step, because these interviews directly related to random call.

Next, we carefully read each transcript to identify each section of text that presented a distinct idea related to random call. When we observed recurring ideas, we created a code and used the code to label these sections of text. Codes categorize sections of text by their content. Creating a code involves summarizing the essence of the idea communicated in the quotes and naming the code using a word or short phrase. Codes primarily emerged from our analysis, but some codes existed a priori, such as accountability as a rationale for using random call. A priori codes came from prior studies of teacher thinking among college biology instructors using active learning (e.g., Auerbach and Andrews, 2018; Auerbach et al., 2018) and the existing literature about costs and benefits of random call. The emergence of these themes was not unexpected; we reasonably anticipated these ideas before conducting this analysis based on prior work. If an idea we anticipated (i.e., an a priori code) proved irrelevant in this data set, we did not use it. Throughout our analysis, we added codes to capture new ideas, removed codes where they no longer made sense, and refined code descriptions to summarize the variation contained in quotes that received the code. When we added new codes, we read through each transcript again to identify any quotes aligned with the new code. When a code contained a handful of quotes, we read all quotes within the code and further refined the boundaries of the code. We did that again for each code after all transcripts were coded. In each step, authors A.H.W. and T.C.A. read transcripts or all quotes within a code and independently determined which codes aligned with which sections of text. They discussed any disagreements until they reached consensus. In rare cases when we could not reach consensus about what code best captured a section of text, no code was applied. We considered the coding process complete when all relevant ideas in the data set had received a code and both researchers agreed about of the application of each code.

Throughout this process, we also engaged in axial coding, which groups related codes together (Charmaz, 2006; Saldaña, 2009). This brings organization and structure to the results by organizing codes into larger themes that are relevant to the research questions (Charmaz, 2006). For example, we had a series of codes related to different aspects of classroom equity that included codes such as "diversity of voices" and "equal representation." We reconsidered these organizations of codes repeatedly in our analysis process by creating visual representations and by soliciting feedback from individuals outside the project about whether the organization of ideas was useful to them. The outcome of axial coding is the themes presented in our results, including both components of random call and rationales for using and not using random call.

Trustworthiness of Qualitative Analysis. There are several attributes of our analysis that make it a trustworthy depiction of how and why the instructors in this study used random call. Trustworthiness in qualitative analysis arises from credibility, dependability, and confirmability (Anfara et al., 2002; Shenton, 2004).

Credibility refers to the precision with which the study design addresses the research questions (Shenton, 2004). Our study aimed to analyze instructors' perspectives of random call. We sought to understand how they use random call and the reasons why they choose to use random call in the first place. Our study design directly aligns with our research questions. We interviewed instructors to specifically target information concerning their rationales and practices related to random call. We also conducted a member check in which we contacted participants to confirm that our coding of their practices aligned with their perceptions of their practices. We emailed a description of their practices to each of the 12 participants and asked 
them: "Is this an accurate description of how you use random call in your course? If not, what revisions need to be made to accurately represent your practice." Eleven participants (92\%) responded to our request for member checking, and all of them agreed that the summaries of their practices built from our qualitative analysis of their interviews accurately depicted their practices.

Qualitative researchers achieve dependability and confirmability by taking measures to ensure that other researchers could reproduce their findings if they carried out the same study in the same context in which the original researchers collected data for their work (Mays and Pope, 2000; Shenton, 2004). We provide a detailed depiction of our methods to strengthen the dependability of our results (Anfara et al., 2002; Shenton, 2004). Confirmability of qualitative analysis requires an unbiased approach and proper measures to protect against bias on behalf of the researchers. We used constant comparison, repeatedly comparing quotes within a code and across codes, to ensure that our thinking remained stable over time. Coding independently first and then discussing to reach consensus protected from individual bias (Anfara et al., 2002). Finally, we provide details about our own positionality to the research. Both T.C.A. and A.H.W. see value in using random call in undergraduate biology courses and also recognize limitations of this practice. Author T.C.A. uses random call as an instructor, and A.H.W. has experienced random call as a student in multiple courses. We carefully designed our data-collection and analysis methods to maintain a neutral position to best serve this report of instructor thinking related to random call (Shenton, 2004).

Identifying Critical Components. After completing our qualitative content analysis, we took additional steps to determine the critical components of random call from the perspective of our participants. Critical components are implementation features that are important to the effectiveness of a strategy. We did not investigate student outcomes in this study. Rather, we aimed to generate hypotheses about what random call components may be critical to student outcomes based on the thinking of experienced random call users. We created a list of all components of random call described by participants from our final codebook. We used two criteria to distinguish which components our participants considered critical: 1) the frequency with which each component was used in our sample and 2) how important participants considered a component to be to their use of random call. We determined frequency by counting how many participants reported using each component. We determined importance using magnitude coding. Magnitude coding involves indicating the intensity or strength of the content you are coding (Saldaña, 2009). We coded the strength of evidence provided by a participant indicating that a specific component was important to his or her use of random call as follows.

We considered all quotes from a participant about a particular component and assigned one of three magnitude codes: no evidence of importance, some evidence of importance, or strong evidence of importance. We considered our data to provide "no evidence of importance" in cases in which a participant described using a random call component without any further indication of the significance of the component. It is critical to note that a lack of evidence of importance does not allow us to conclude that a participant considers a component unimport- ant, as we did not specifically ask participants to describe the importance of each component of their random call practice.

We considered our data to provide "strong evidence of importance" when participants indicated that they "always" implement that component, are "careful" to use that component, "never" omit that component, or otherwise indicated the critical nature of a component. If a participant specifically mentioned a practice when asked to provide boiled-down advice to an instructor new to random call or if he or she provided a detailed rationale for including a component, we considered this strong evidence of importance. We also considered it strong evidence of importance if a participant described omitting a random call component and later adding the component back in because its omission negatively affected his or her practice.

We considered our data to provide "some evidence of importance" when participants did more than mention their use of a practice, but failed to meet the requirements for strong evidence.

We distinguished between components hypothesized to be critical and other components by considering data on both the number of participants who used the practice and evidence that participants considered a practice to be important to their implementation of random call. Hypothesized critical components were those:

1. used by more than half of participants in the study, and

2. for which more than half of those who used the component provided strong evidence of importance.

\section{RESULTS}

We rely heavily on quotations from participants to support our results. The selected quotations represent ideas expressed by more than one participant, unless otherwise indicated. Pseudonyms distinguish each participant. We represent the gender of participants using traditionally male and female names to maintain important aspects of their identity while protecting sensitive information. Some quotes are lightly edited for grammar and syntax, and we use ellipses to denote omitted sections.

\section{Research Question 1: How Do Random Call Users Weigh Benefits and Costs in Making Decisions to Use Random Call?}

Random call users perceived both costs and benefits of implementing random call as an instructional strategy. These echo results from prior research (Table 2).

We highlight three ways in which random call users weighed perceived costs and benefits. Our presentation of these themes below is interwoven, because participants considered them together.

- Random call users weighed the diversity of voices benefit of random call heavily in their decision making.

- Random call users saw student anxiety as a potential cost of random call, but thought this cost could be substantially reduced through specific components of their random call implementation.

- Some random call users saw a small amount of anxiety as a benefit to students. 
TABLE 2. Perceptions of costs and benefits of using random call in large undergraduate biology courses among random call users

Random call may increase students' accountability to work.

Random call may encourage students
to complete preclass work, work
during class time, and engage in
higher-quality discussions with
peers.

"I know that the students will learn more if they talk to each other. They will get a chance to think and explain. If they just sit and listen, or if they work individually on things, they're missing an opportunity to think about the content with somebody else who is thinking about it maybe a little bit differently ... I think, in a big classroom setting, there's really no other way to get students to do that kind of work unless you call on them sometimes to make sure they are not just goofing around or zoning out or whatever."-Rebecca

\begin{tabular}{|c|c|}
\hline \multicolumn{2}{|r|}{ Random call may lead to greater diversity in the voices heard in class. } \\
\hline $\begin{array}{l}\text { Random call may ensure that students } \\
\text { who speak are representative of } \\
\text { the students in the course and } \\
\text { work against instructor implicit } \\
\text { biases. }\end{array}$ & $\begin{array}{l}\text { "I know, like everybody else, that there are a limited number of people who will volunteer and that } \\
\text { limited number of people is not representative of the people in my classroom. And so, I [use } \\
\text { random call] for equity of voices in the classroom."--Renee }\end{array}$ \\
\hline $\begin{array}{l}\text { Having a voice in class may build } \\
\text { students' confidence and foster a } \\
\text { sense of belonging. }\end{array}$ & $\begin{array}{l}\text { "It's really important to me that all students are engaged in the material and particularly students who } \\
\text { are educationally disadvantaged, they already have a leg down, so to speak, on their peers. So it's } \\
\text { really important to me that I hear from them too. We say 'underrepresented students' but that } \\
\text { means underrepresented in STEM and I believe that all students, all people, belong in STEM if they } \\
\text { want to and, once their voices are heard, it's more likely that they'll feel like they belong."-Rachel }\end{array}$ \\
\hline $\begin{array}{l}\text { Diversity in who speaks during class } \\
\text { may improve learning by exposing } \\
\text { students to more diverse ideas. }\end{array}$ & $\begin{array}{l}\text { "If you just allow [volunteers], you're going to keep on hearing from the same people which means } \\
\text { you're going to keep on hearing from the same perspectives. You're getting a richer, more full, more } \\
\text { representative discussion if you are encouraging and forcing those other perspectives to come } \\
\text { out."-Robin }\end{array}$ \\
\hline \multicolumn{2}{|r|}{ Random call may increase student anxiety. } \\
\hline $\begin{array}{l}\text { Random call may contribute to } \\
\text { anxiety among students and may } \\
\text { be particularly acute for some } \\
\text { students. }\end{array}$ & $\begin{array}{l}\text { "In my youth, I never, ever would have raised my hand or answered a question so I'm really sensitive to } \\
\text { the fact that there are many students who are like me, who may know the answer, but they don't } \\
\text { want to say it out loud. So that's why I use group [random call] rather than [individual random } \\
\text { call] because I feel like for some students ... I mean they'll say 'I'm not coming to class. If there is a } \\
\text { chance that I will have to speak out loud, I'm just going to go. I'm absolutely nervous about having } \\
\text { to speak aloud in front of people."'-Ramona }\end{array}$ \\
\hline
\end{tabular}

Random call users often described improving equity in their classrooms as a major motivation for using random call. In fact, some felt obligated to use random call to achieve greater diversity of voices in their courses. They weighed this benefit of random call so heavily that it outweighed their serious concerns about how random call had the potential to make students feel. Rita described how she initially made the decision to use random call and her concern about student anxiety,

I haven't done random call always in my classes. But I had read an article and it was probably Sarah Eddy's article on equity in the classroom and equity of voice. [I] got really annoyed with myself that when I was asking questions, I was often calling on the very first hand that went up, which was that same guy usually in the front row and I was just-every time I was really annoyed. I was like "Dang it! I called on that guy again." So, I decided to start [using random call] just to force myself to get more voice in the classroom and different voices, but I was worried about it because I know, as a student, I hated random call. My horror was to get called on in a class. So I wanted to be really respectful about it and I wanted to do it in a way that was supportive.

Random call users addressed their concerns about student anxiety by developing components of random call that they expected to reduce student anxiety. We describe these components in depth in the next section (Research Question 2). Regina explained how she struggled with the decision to use random call due to anxiety concerns, and how she implements random call in specific ways in order to reduce the anxiety it can cause students,

[Using random call] took me awhile to be convinced of ... the more I thought about it, the more I thought, "Yeah, I'm not helping with stereotypes and inclusiveness and why am I not doing it?" I know it feels hard for me because it's hard for them, and I empathize with them. So, I had to find a way that I felt comfortable with it. I sort of set up a contract early on in the class with [students]. I tell them, "I'm never going to random call an individual person. I'm only going to random call groups and you're reporting for what's around, and I'm never going to random call a group if you haven't had a chance to think about it first with each other." So I think that helps. I think the majority of students are okay reporting out for a group.

Some random call users thought most student anxiety was mitigated with careful implementation, and others expected that some anxiety remained. However, the latter group felt that the benefit of increased diversity of voices outweighed the cost of the remaining anxiety.

A few random call users explained that a little anxiety could actually benefit students, because it could be a "motivator ... that can help you to perform better" and because dealing with some anxiety in a classroom context could help students learn to deal with it elsewhere. Robert took many steps to reduce student anxiety, but he also thought some anxiety could contribute to students' professional development, 
Anxiety is interesting. The root emotion in anxiety is fear. And what seems to be coming out of the literature is that people who get over anxiety are less prone to it if they've experienced many small stresses. So very large stresses are bad, even traumatic or life-destroying. But many small stresses can be productive.... I see random call as one of those little stresses; you can grow from this.

\section{Research Question 2: How Do Instructors Implement Random Call in the Classroom?}

Each participant in this study met our general criteria of employing "a randomized approach to select a student or group of students to share their thinking with the whole class," but the specific components of the random call implementations varied. Here, we present the components that were part of how our participants used random call. We organized the components chronologically to reflect and emphasize the sequence of steps through which instructors progress when carrying out a random call (Figure 1). The first two components take place early in a course to prepare for random call. The next components constitute a single enactment of making a random call and are divided into three parts: pose a question, make a selection, and hear from students (Figure 1). The last component deals with repeating random call throughout the term.

Within this chronological sequence, we highlight the components of implementing random call that our participants considered critical (dark gray boxes in Figure 1). Table 3 provides counts of the number of participants who reported using each component and the proportion of users who indicated that this component was important to their practices.

In addition to providing details about how participants implemented random call, we summarize the reasoning participants provided for using a component. These align with participants' rationales for selecting random call as an instructional strategy, including reducing anxiety, contributing to accountability, and increasing the diversity of voices heard. We indicate that a rationale was associated with a component in Figure 1 using colored borders if one or more participants provided this rationale for the component. We use bolded text in the following sections to bring attention to the rationales given for each component and to facilitate making links between Figure 1 and the text.

\section{Preparation}

Use Random Call Early in the Term. Instructors began using random call within the first 2 weeks of a course and often introduced the practice on the first day. Participants explained that using random call "right off the bat" helped to "set up the tone of course" and demonstrate how the course would be run. Ramona described a semester when she had not started the semester using random call,

It didn't go well. You'd almost need a new instructor to come in, take over for a little bit, and then come back and start back over again [...] If I start a semester [by introducing random call] and they know it's going to happen, then they're really good about it. If I start [random call] later, they seem really resistant to talking in front of class.

Explain to Students Why You Are Using Random Call (Hypothesized Critical Component). Random call users commonly took
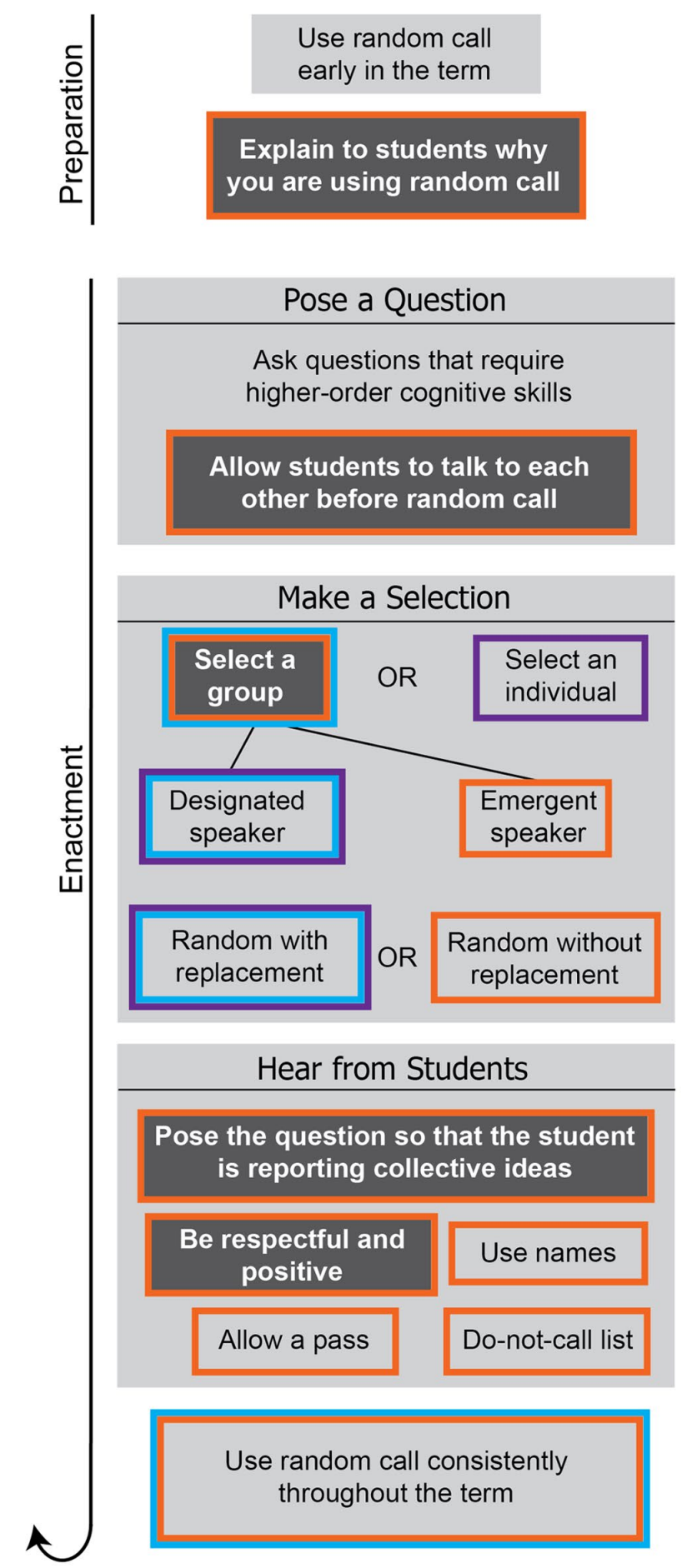

Critical component $\square$ Anxiety-reducing $\square$ Accountability $\square$ Diverse voices

FIGURE 1. Components used by our participants in preparing for and enacting random call. Components hypothesized to be critical to effective use of random call, based on the thinking of our participants, are represented in dark gray boxes. Investigation of student outcomes will be necessary to test these hypotheses. The rationale for using a component is represented with colored borders. We included a rationale in this figure if it was described by at least one participant as influencing the decision to use a component. 
TABLE 3. Counts of participants who used each random call component and the percent of component users for whom the data provide strong evidence that they consider the component important to their random call practices

\begin{tabular}{|c|c|c|}
\hline Random call component $^{\mathrm{a}}$ & Count $^{\mathrm{b}}$ & $\begin{array}{l}\% \text { of users with } \\
\text { strong evidence of } \\
\text { importance }\end{array}$ \\
\hline Use random call early in the term & 11 & 18 \\
\hline Ask questions that require higher-order cognitive skills & 4 & 25 \\
\hline Allow students to talk to one another before random call & 9 & 67 \\
\hline Designated speaker & 3 & 33 \\
\hline Emergent speaker & 7 & 14 \\
\hline Random with replacement & 3 & 33 \\
\hline Random without replacement & 10 & 10 \\
\hline Pose a question so that the student is reporting collective ideas & 9 & 56 \\
\hline Use random call consistently throughout the term & 11 & 45 \\
\hline
\end{tabular}

${ }^{a}$ Hypothesized critical components shown in gray.

${ }^{\mathrm{b}}$ All counts are out of 12 random call users.

time to explain to their students why they used random call. Most instructors did this verbally in class, but some included a justification for random call in the syllabus as well. Some participants anticipated that explaining why they were using random call would help to reduce students' anxieties (Figure 1). For instance, Rebecca offered this advice to someone new to using random call,

Don't just try to do it without saying why you're doing it and reassuring them that it's okay to be wrong. Don't do it that way because it'll fail or it'll make them really anxious. Think about your reasons for doing it. Then tell them why you're doing it.

Instructors' explanations to students emphasized the benefits of random call, clarifying that they "would like to hear from everybody" and that random call is "in service of your learning." For example, Regina demonstrated for students how few of them would volunteer to answer a question and then explained that "[asking for volunteers] is not a technique I'm going to use often," because if she did then she was "not going to hear from most of you." Ramona modeled how she helped students understand why she was using random call and "why it will be beneficial for them":

I want you to share because there very often are some students who are still confused and they may understand the way you're describing it a lot better than I am. I also often say sometimes the way I'm asking questions might be confusing, so you might be able to help me understand better how to put the words to this or explain it ... The idea is I'm not quizzing you. You are helping me. You're helping the other students by giving this information.

\section{Enactment: Pose a Question}

Ask Questions That Require Higher-Order Cognitive Skills. The first step of enacting random call in a class session is posing a question to students. Some participants explained that they tended to reserve random call for questions that warranted more discussion among students and for which students may have used "different problem-solving strategies" to come up with an answer. Robert said,

We try to emphasize stuff that's more synthetic or interpreting data or issues of experimental design ... synthetic in the sense of students pulling a bunch of ideas together where they need to talk about it and work through, as they would with a short answer on an exam.

Renee reported that, since she started teaching, she had shifted the types of questions that she considered worth spending time on in class. For her, questions that are worth class time involve students

applying their knowledge to problems in the course. Because if you do random call, [students] have to have a good amount of time to discuss it, like one minute, two minutes. They have to have time to talk about it, especially depending on the level of the question. If I'm going to spend two minutes of talking, plus the report out on something, it better be worth it, it better be practice, it better be application. So I've shifted the kinds of questions that I ask to more high-level Blooms only.

Allow Students to Talk to One Another before Random Call (Hypothesized Critical Component). Most participants provided students with a period of time to discuss a question with their peers before selecting someone to report out to the class, whether they called on individuals or groups. Many indicated they always included this random call component. Participants reported that allowing peer discussion before asking someone to respond in front of the class helped relieve student anxiety (Figure 1) and "avoid the deer in the headlights moments." Rita explained, 
So, I wanted to do it in a way that allowed people to confer with their neighbors to make sure that they felt confident about what they were about to say, which is why I do that. I always [allow students to talk in groups] before I would call on somebody.

Similarly, Robin described how time to work in small groups allows students to prepare their reasoning before they share it in front of the whole class:

So, usually when I'm wanting to have a student voice, I'll first let them know that I'll be calling on a student or a group of students. So, they have time to work on those problems and then prepare what they would report back to the larger group. I'll say, "We're going to spend 10 minutes working in this practice problem and then I want us to share out in a large debrief so we're all in the same page. We can answer your questions about it." So, I like to do that so they know what's coming. They kind of have that preparation in mind of what they would end up saying and they're writing it down kind of to summarize what their discussion or what their process or what their answer is.

Enactment: Make a Random Call. After posing the question to the class and allowing students time to formulate and discuss their thinking with their peers, the next step in the sequence is making a randomized selection. We differentiate this into several components related to the unit of selection and the randomization procedure.

Select a Group (Hypothesized Critical Component). Instructors who selected groups assigned a number to each group or asked groups to create a group name. Both approaches provided a simple identifier to randomize and announce to the class. Random call users selected groups by drawing a note card with a group name, calling a group from a randomized list, by rolling "an $N$-sided die, where $N$ is the number of tables in the classroom" and each table is a group, or by using a random number generator. Those who selected groups were likely to see choosing groups as an important component of their implementations of random call (Table 3). Most commonly, these instructors felt that calling on groups lessened student anxiety compared with calling on individuals. Rhonda described how she had cotaught a course that used individual random call and had since decided only to use group random call in her courses:

The students got nervous, like they were really scared that they were going to be called on. Some of them even said that they sat there the whole class worrying about being called out.... I think as a group they feel more like, well even if they're wrong, "all four of us were wrong, so it wasn't just me." It's more that they know they're not the only one who is responsible for the answer, I think.

One instructor indicated that calling on groups improved student's sense of accountability, because she could call on each group more times per semester than she could call on individuals (Figure 1). In classes with $300+$ students, she had not been able to call on students more than once per semester, so students knew "they're not going to be called again." Putting students in groups allowed her to call on each group multiple times per semester.

Whether calling on groups or on individuals, instructors in a large classroom needed to know where the selected group or student was located. Participants solved this problem in a few different ways, such as creating a classroom map of group locations or asking students to indicate their location on a note card. Renee explained how she did not have trouble finding groups in her 100- and 300-student courses, but she had to adopt a new strategy for a 600-student course. In each of her courses, students created a group index card that listed a group name they had created and the names of the individual students in the group. She randomly selected groups by choosing an index card,

In the 600-person classroom, by calling a group name, no one person felt on the spot and I didn't know where they were. And so they were less likely to answer; they were more likely to ghost me in my big class. What I'm doing now is I'm having them put their location on the card. "Are you in part of the room 1, 2, 3, 4, or you're in the front or in the back?" And it's going really well because I can say, "Oh, it's the Bio Buddies. There you are, okay." They tell me their location, I look up there, and they answer me because they're like "Yeah, that's me."

Instructors who call on groups have the additional step of determining which student in a group will speak on behalf of the group.

Emergent Speaker. Some instructors allow this decision to be made among the group members. Often these participants thought that this would relieve some anxiety for students by allowing students who are more outspoken or more confident in their responses to represent the group (Figure 1). Renee mentioned that allowing a group representative to be chosen by group mates could help more anxious students feel comfortable,

Not only does [group random call] help me because I get to go through the list faster, there's less anxiety, presumably for having a group to rely on. Because if some person in your group gets it started, you can be like, "Well, anybody can answer. It doesn't have to be you."

Designated Speaker. Other instructors had a hand in designating which student would speak on behalf of the group. Some instructors asked students to assign roles within their groups, including the role of a reporter who was responsible for sharing the thinking of the group with the class. Participants generally did not describe strategies for ensuring that these roles rotated within the group from class session to class session. Others selected a student from the group by name. Participants thought designating the speaker would include students who may not otherwise participate and thereby increase the diversity of voices heard in class (Figure 1). They described targeting a student who was not a "fast responder" or who "hasn't been speaking up."

Regina designated the group reporter for a class session by declaring that the reporter for the day was the person who met a certain neutral criterion for the day. For example, the criterion 
for the day might be the person who went to bed the earliest the night before or the person who ate pizza most recently. She explained that adding this structure to designate a speaker for each group improved accountability and the diversity of voices heard in class:

I really believe the more structure you bring to any part of your course, the more inclusive it will be. And too many times, especially when I first started teaching in this way, I just assumed students know how to interact with each other, and they don't. And there's power dynamics in a small group. There are underrepresented people in a small group, like all these things are there and they're with people they don't necessarily know, but they need to build trust with. So, the reporter-I don't do this on every question and they've seen it throughout the semester. They've been structured in different ways but it just helps to make sure the loudmouth isn't always the loudmouth and the introvert is forced out of their comfort zone a little bit. It's about accountability, but a little bit of professional skills development.

It was common for instructors to designate a speaker in some instances and allow the speaker to be emergent in other cases.

Select an Individual. Some random call users selected individuals rather than groups. Instructors who selected individuals announced the student's name to the class. At least one participant felt that selecting individuals was "preferable" to selecting groups, because it ensured a greater diversity of voices (Figure 1). This participant, Robin, worried that calling on groups allowed some students to dominate class discussions,

Other times, I want to have individual students speak up. And I think that that is even preferable because it's calling, it's allowing a specific student a chance to speak out. So, if I'm calling on a table, there could be up to seven students at the table so I still have the situation where maybe there's a dominant voice at that table and it will always be that student who ends up representing a group.

Robin seems to be thinking that calling on groups without designating a speaker could reduce the diversity benefits, because not all students are equally likely to be heard in the classroom. Other instructors, including Ramona, valued a student being able to avoid having to speak in front of the class due to group random call:

I use group rather than [individual random call] because I feel like for some students it's just-I mean they'll say "I'm not coming to class if there is even a chance that I will have to speak out loud, I'm just not going to go. I'm absolutely nervous about having to speak aloud in front of people."... I always say in the beginning, "You know there's somebody in your group who is like a theater major or just loves talking so let's let them be that person. The rest of us will just give them some ideas."

The contrasting viewpoints of Robin and Ramona highlight how instructors weigh costs and benefits of specific components of random call in light of their rationale for using the practice and their students.
Random with Replacement. A random selection with replacement occurs when a selected unit (i.e., individual or group) is put back into the pool immediately after selection (Lohr, 1999). In random call with replacement, a student could be selected more than once in a class period. Instructors using random call with replacement opted to roll a die, shuffle a pile of cards with student or group names, or use a random number generator. Ruth thought that the fact that a group's number could appear again on the next roll kept students accountable and achieved a wider diversity of voices in the discussion,

People don't have the feeling they can answer once and now, they don't have to answer for the rest of the period or that week because there is some sort of a pattern that goes around ... it's random. Also, we all have biases, so I wanted to correct my own biases and also remind myself, well, I should encourage some of the people that are a little culturally less inclined to answer right away.

Random without Replacement. A majority of participants made randomized selections without replacement. This means that they removed students from the selection pool after they had been called. Instructors using random call without replacement often had a randomized list of student or group names or a stack of cards with names that they did not shuffle until they had gone through every card. They crossed off a name after calling it and continued through the list. After everyone had been called once, they repeated the process with the same list or a newly randomized list. Most instructors only made it through the list or stack of cards a few times each semester. For the most part, participants did not provide a specific rationale for selecting without replacement.

Rita expected that using random call without replacement relieved some of the anxiety for students, because they knew that they would not be called on again any time soon:

I know that I would have felt great relief in knowing that that once I was called and I was done, that was it for the semester. I wouldn't have to deal with that again.

This is an example of a random call user placing greater value on reducing the cost of anxiety than on the accountability benefit of random call. Her approach would result in students knowing that they would not be accountable for speaking in front of the class after they had done it once.

Enactment: Hear from Students. After posing a question and making a selection, instructors hear from students. Random call users described several components of their implementation of random call that take place while they are hearing from students, including two components they considered critical.

Pose a Question so That the Student Is Reporting Collective Ideas (Hypothesized Critical Component). Participants carefully worded their questions to inform students that they were responsible for reporting what their group discussed rather than reporting their own ideas. Both instructors who called on individuals and instructors who called on groups used this practice. In some courses, groups had been formally assembled, 
and in others, students discussed the question with nearby students. Participants, including Rachel, indicated that they intentionally used certain prompts to indicate to the class and the speaker that the speaker was sharing on behalf of a group of students:

I'll ask for somebody randomly to share what they and their group have talked about. I often try to frame it as what you and your partners have been talking about to kind of take some of the pressure of random call off students.

Instructors reported that framing the question this way to the speaker reduced student anxiety (Figure 1). Rita described it this way:

I tell them that I've got their name on note cards, that I'm going to be pulling the note card and I'm going to be asking that student to respond, but they'll always have a chance to talk with their group. That, if they choose, when they answer, they can say "My group and I..." They don't have to individually own the response. For some students, that makes them feel a lot less nervous if they give the response on behalf of the group and not just on their own behalf.

Be Respectful and Positive (Hypothesized Critical Component). Random call users commonly viewed kindness, respect for students, and praise as a component of their implementations of random call and reported that this approach went a long way toward reducing student anxiety with random call (Figure 1). Participants explained that they were "very supportive," "really friendly," and "extremely positive" when students shared their thinking with the class. Some also discussed how they "praise students on their effort, not on the correct answer" and "always thank students for participating [because] this is helping us all learn."

Some participants indicated that creating a classroom environment in which students trusted the instructor to do what was best for them was a pedagogical goal of theirs that extended beyond random call. For example, Rebecca spoke about creating an inviting classroom by connecting with her students on an interpersonal level:

I just try to be really understanding like, 'I know you guys are having a hard week. I know the exam was hard. I know this. I'm here to help you.' I just try to remind them that I'm not out to get them, and that my goal is-I would be thrilled if they all got A's in the class. I'm not there to penalize them or be punitive. So, I think there's a lot of-I do a lot of instructor talk around helping them, making them feel comfortable, laughing, making jokes, being whatever, just trying to be nice. This is a nice environment. I want you guys to be happy here.

Similarly, Renee talked about the necessity of building a sense of community in an active-learning classroom where students will be asked to work and to share their thinking:

The community that you build in your classroom for active learning is actually really, really important, right, because you're going to asking people to take risk, to be wrong, right? That's what it is and you need to have a safe space to them to do that, and so-build a community.
Use Names. Some random call users reported that using student or group names during random call helped them connect with their students and reduce student anxiety (Figure 1). They also saw using names during random call as serving a larger purpose of helping them build a relationship with their students. Rose explained,

It serves several purposes. This is the way I make sure I learn their names, first of all. And it also means when I'm calling on students, I'm calling them by name instead of like, "Hey, you!" And I find they are very receptive, they're very appreciative of a professor knowing their name especially when it's a large class. So, that really, really helps.

Robin explained how she tried hard to make sure she was pronouncing students' names correctly,

If you're calling a student by name, you have to be respectful enough to do it right. I've had students be like "Oh, yeah. Everybody gets my name wrong." But that kind of defeats part of the purpose. I guess this goes back to why you use random call at all. It forces you to get to know your students on an individual level.

Participants described a few strategies that helped them pronounce students' names, including practicing before class and asking students to write phonetic descriptions of their names on a note card.

Allow a Pass. Some participants explained that, in order to reduce student anxiety, they allowed students to pass to the next person or group when they felt unprepared to contribute (Figure 1). Participants generally saw this as a rare occurrence, and most did not formally announce to students that they would allow a pass. Robert explained that he always allowed students to pass and never shamed them for doing so. He also indicated that, if passing became too common, he would reiterate to students that participating in class discussion represented an important part of the course and would ultimately benefit their development,

I try never to scold students for passing. In fact, I thank them for speaking up and all that. But if I get five or six [passes], sometimes I'll just stop and say, "You guys, I'll honor your passes, that's fine, but I just want to remind you, you want to be working in a clinical setting or be running a company 10 years from now or directing research projects and when you're in clinic and your attending physician comes up and says, 'What should do we do here?,' you got to say ... A little reminder, you guys. You can't do this the rest of your life."

Do-Not-Call List. A few participants, all of whom taught at the same institution, implemented a policy whereby students could ask to be placed on a formal do-not-call list if they did not want to ever be randomly called on. Though participants who instituted a do-not-call list stated they would "always honor" these requests, they saw this policy as accommodating particularly extreme cases of student anxiety. These instructors had other components in place to reduce anxiety for the majority of students. 
Use Random Call Consistently throughout the Term. Participants used random call consistently throughout the course. Participants varied in how frequently they used random call, with some using it 15 or more times per class session and others using it once per week. Some participants thought using random call consistently throughout a semester could help reduce student anxiety (Figure 1). For example, Rita indicated that people "aren't as nervous" when they "know what to expect." She said,

Anxiety is all about anticipation of something that could happen, right? Well, if you [use random call] enough in the class and the students know it's going to happen, then they don't need to anticipate it because it's going to happen ... so I do get frustrated with myself when I don't do random call on a regular basis because I do feel like if I'm going to do it, I should be doing it in every single class period so that they never forget about it and they just get used to it.

In a related vein, Renee explained that it was easier and more efficient for her, as the instructor, if she always used random call, rather than a combination of using random call and asking for volunteers. Renee found using random call each time she wanted a student to share his or her thinking with the class simplified the decisions she had to make while teaching,

What was so hard for me before is ... I didn't know whether to ask for a random call or a volunteer. Whether to go to my [randomized] list or just take a volunteer took up so much of my mental head space when I first started teaching, because I didn't have the idea that I would always do random call every single time. I didn't have that rule in my head or anything. And so I was making a decision each time.

One participant explained that consistently using random call helped ensure that students remain actively engaged even when she was not able to directly monitor their engagement. Regina thought that using random call at least once a day maintained an adequate level of student accountability (Figure 1):

I need to use [random call] at least once a class, preferably a few times during class, because it sets up that accountability, especially for different parts of the room where I might not be roaming.

\section{DISCUSSION}

This work provides new insights into the details of how random call is implemented in real classrooms and the rationales underlying users' implementation decisions. Figure 1 provides a framework of components that can be part of implementing random call. Instructors can draw on this framework as a representation of how others use the practice. Researchers can use this framework to carefully articulate how random call is used in the classrooms they study. A detailed description of what we mean when we say "random call" is crucial to investigating how random call influences students.

\section{Next Steps: Investigating How Random Call Influences Different Groups of Students}

Our work reveals instructor perspectives about random call, but does not investigate student outcomes or perspectives. The par- ticipants in our study made reasonable assumptions about the effects of random call on their students, often based on years of teaching experience and sometimes drawing on their own experiences as students and the research literature. Unfortunately, the existing literature falls short of identifying specific components of random call and illuminating the effects of these components on different groups of students. The thinking of our participants aligns with other work indicating that instructors place high value on their own experiences as they make instructional decisions (e.g., Andrews and Lemons, 2015). Yet, it was not uncommon for our participants to mention specific research that informed their decisions to use random call, and we expect many would be highly receptive to new research that more deeply explores the impacts of random call.

The costs and benefits our participants attributed to random call and to specific components of random call generate hypotheses that can be thoroughly investigated with student populations. Future work needs to examine the details of random call practice (i.e., which components are used) and how they influence various outcomes for students (e.g., learning, confidence, belonging, anxiety). Critically, this work must be done in different instructional contexts and for different groups of students, because the effect of random call may not be same for all students. The framework generated by this work will help researchers take these next steps.

Speaking in front of the class is a social interaction that can bring a student's social identity into the spotlight. Students with a minority social identity may experience social interactions in the classroom differently than students with a majority social identity. For example, compared with white students, Asian-American, international, and historically underrepresented minority students preferred to have ideas explained to them by peers in small-group discussions rather than explaining ideas to their peers (Eddy et al., 2015). This may mean that these students are less likely to talk in small-group work and would be less likely to organically emerge as a speaker for a group. If that is the case, then randomly selecting groups and allowing the speaker to organically emerge would not actually achieve greater diversity of voices in the classroom. Other work indicates that Black science students may feel enormous pressure to represent Black students well in science courses, because they are aware of negative stereotypes (Julie Stanton, personal communication). We might hypothesize, then, that Black students experience increased anxiety compared with majority students when asked to speak in front of the class, because they perceive the consequences of having their science knowledge negatively evaluated to be much higher. In another study, students who identified as part of the LGBTQIA spectrum (lesbian, gay, bisexual, transgender, queer, intersex, asexual) indicated that active-learning classrooms increase the relevance of their LGBTQIA social identities, creating both challenges and opportunities (Cooper and Brownell, 2016).

Though random call can increase the diversity of voices in the classroom (e.g., Martin et al., 2006; Eddy et al., 2014), it is unclear whether it creates equitable classroom experiences for students and for whom the benefits outweigh the costs. Currently, we lack evidence of how racial, ethnic, gender, LGBTQIA, disability status, and other social identities influence the experiences of random call for students. The participants in our study describe a variety of components they enact specifically to 
mitigate anxiety (Figure 1), and some of these approaches may address potential differences in student experience that result from social identities. We encourage researchers to pursue studies that specifically indicate the components that make up the random call practice investigated and that disaggregate outcomes based on students' social identities. Eddy and Hogan (2014) provide a strong model for carefully investigating the impact of instructional practices on different groups of students. Large studies can also recognize the intersectionality of social identities and examine the impacts of random call on more specific groups, such as Black men or women who identify as part of the LGBTQIA spectrum (e.g., McCall, 2008). Expanding our knowledge about the potential differential impact of random call will help instructors make decisions that weigh their instructional needs, context, and student body.

It will also be important for future studies to examine how much anxiety is too much in active-learning classrooms. Some researchers have suggested that the Yerkes-Dodson law may be relevant to considering the effects of student anxiety in undergraduate STEM classrooms. This law states that anxiety and performance have a curvilinear relationship, in which both low and high levels of anxiety are associated with poorer performance more than intermediate levels of anxiety (Yerkes and Dodson, 1908; Keeley et al., 2008; England et al., 2019). The participants in our study perceived that they could mitigate much of the anxiety associated with random call for their students. Future work is necessary to determine if that is true, and if it is true, whether they achieved a level of anxiety that motivates and supports students to perform, rather than hindering their performance? And how does that differ among students?

\section{Our Results and Other Relevant Research: Hypothesized Critical Components of Random Call}

There is very little prior work regarding how random call could be implemented to maximize student outcomes. In fact, we are aware of only one research group that has investigated specific components of random call. Elise Dallimore and colleagues have considered the use of random call in business and accounting courses and have made recommendations about how to implement random call. These recommendations emerge from studies investigating the impact of random call on students, faculty panels asked to generate key practices for making random call less intimidating, and student recommendations about instructor behaviors that promote effective discussion. Notably, the recommendations offered by Dallimore and colleagues align well with some of the critical components suggested by our participants. These recommendations include explaining the use of random call to students, using random call each class session starting on the first day of class, giving students time to think and prepare before using random call, asking open-ended questions with multiple possible reasonable responses, and providing positive and constructive responses to what students say in whole-class discussions (Dallimore et al., 2004a,b, 2013).

Research related to other teaching practices can also be useful in hypothesizing about critical components of random call. We briefly review prior research that is relevant to the critical components of random call identified by our participants. We also discuss random call components that did not meet the criteria for critical components in this study, but could be hypothesized to be important based on other research.
Explain Why You Are Using Random Call. Participants viewed explaining the rationale for using random call as an important strategy for setting up the practice and for managing student anxiety (Figure 1). Explaining pedagogical choices is one of five categories of noncontent instructor talk identified in student-centered college courses (Seidel et al., 2015). Noncontent instructor talk contributes to creating a learning environment and can influence student motivation, resistance, and self-efficacy (Seidel et al., 2015). Being explicit with students about the reasoning behind pedagogical choices throughout a semester may be an important strategy for preventing student resistance to evidence-based teaching strategies (Seidel and Tanner, 2013). This approach can help students see the value in what they are asked to do and communicates that the instructor respects students enough to share his or her decision making. In addition, the task of articulating reasoning for random call provides an opportunity for instructors to think carefully about their rationales and the alignment between rationale and practice. Furthermore, if part of an instructor's rationale for using random call is increasing the diversity of voices in the classroom, explaining this rationale is an opportunity to communicate to students that equity is an explicit goal of the instructor, that student thinking is valued by the instructor, and that the course does not aim to "weed out" certain students (Tanner, 2013). This transparency into instructor thinking may be particularly important for framing practices that have the potential to be viewed negatively by students, such as random call. Student buy-in to active-learning practices may contribute to their successful engagement in these practices and, ultimately, the outcomes they are able to achieve (e.g., Cavanagh et al., 2016). Explaining our reasons for using particular strategies may be important to fostering this buy-in. Despite prior work suggesting the importance of explaining to students the rationale behind using random call, we currently lack evidence about the impact of this practice.

Allow Students to Talk to One Another before Random Call. Participants thought that allowing students to talk to one another reduced anxiety associated with random call (Figure 1). Participants also saw random call as a potentially important strategy for holding students accountable for engaging in meaningful discussion when asked to do so in small groups. The role of peer discussion in reducing anxiety associated with random call has not been empirically investigated, but there is strong support for the learning benefits of students discussing a question or problem with peers before a larger class discussion or instructor debrief. Students explaining their reasoning to one another is a critical component of effective peer instruction and possibly active-learning more generally, provided that students are asked sufficiently challenging questions (e.g., Eddy et al., 2015; Vickrey et al., 2015). Peer discussion can contribute to student learning even if no one in the group initially knows the answer to a question (e.g., Smith et al., 2009). Discussing reasoning with peers can also improve understanding in students who could initially answer a question correctly (Brooks and Koretsky, 2011). Importantly, students are more likely to exchange evidence-based reasoning in small-group discussions when they are specifically prompted share their reasoning with one another (Knight et al., 2013). Future work will need to investigate how talking in groups changes students' experiences 
of random call, especially the anxiety they feel about being asked to speak in front of the class.

Select a Group. Participants who randomly selected groups believed that this helped reduce anxiety compared with calling on individuals (Figure 1). Participants disagreed about whether calling on individuals or on groups was a better approach for making students feel accountable and also had differing ideas about how calling on groups affected the diversity of voices heard in the class. Existing research has not investigated the effect of randomly selecting groups on learning, anxiety, diversity of voices, equity, or accountability. Given the increased use of small-group work in undergraduate STEM courses, investigating how groups can be used to improve students' experiences and outcomes with random call and beyond is an important avenue for future research.

Pose the Question so That the Student Is Reporting Collective Ideas. This practice was important to participants, with several indicating they "always" prompt students to speak on behalf of the peers with whom they discussed the question. This component is specific to instances when students speak in front of the class and has not been the focus of prior research. Future research can reveal whether students' anxiety is eased by knowing they are representing the thinking of a group rather than only their own ideas. Fear of negative evaluation accompanies random call for some students, but this may be mostly focused on being evaluated as an individual (Cooper et al., 2018). A course in which random call is always framed as collecting group contributions, rather than individuals' ideas, could help ease fear of negative evaluation. This might be an especially important strategy for students who feel the pressure of representing their social identities positively, which may be true for students from groups historically underrepresented in STEM.

Be Respectful and Positive. Participants indicated that treating students with respect (i.e., as the instructor would wish to be treated) and responding positively to their contributions created an environment that made random call less threatening to students. Participants described these behaviors as very deliberate decisions that they made, rather than as natural manifestations of their personalities. Thus, it aligns with our data to consider this part of a teaching practice, rather than a style or personal attribute. We propose that instructors can improve their use of this component in the same way that they can practice and improve the implementation of other components of random call.

The precise effect of being respectful and positive while using random call warrants further investigation, but prior work indicates this practice can be important to student outcomes. Being respectful and positive toward students is part of instructor immediacy, which encompasses instructor behaviors that decrease the social distance between instructors and their students (e.g., Mehrabian, 1971; Seidel and Tanner, 2013). Instructor immediacy can reduce student resistance toward and increase student participation in a teaching strategy, increase student motivation, and even positively impact learning (Seidel and Tanner, 2013).

Recent empirical and theoretical work indicates an important role for kindness and trust in STEM higher education environments (e.g., Estrada et al., 2018, 2019). Kindness cues, which are "cues affirming social inclusion and respect for dignity" (Estrada et al., 2018, p. 263), may help counter the negative consequences for students of cold, unwelcoming, and implicitly or overtly prejudiced environments sometimes characteristic of STEM education (Estrada et al., 2018). Importantly, kindness cues can increase students' sense of integration in the scientific community, ultimately contributing to the intention to persist in STEM (Estrada et al., 2019). Additionally, students trusting their instructors may positively influence student engagement, commitment to active-learning strategies, and course outcomes (e.g., Cavanagh et al., 2018), and the benefits of trusting one's instructor may be especially pronounced for some groups of students, such as Black males (McClain and Cokley, 2017). Though these studies have not specifically studied random call, they suggest that our participants' perceptions about the importance of kindness to their implementations of random call are aligned with social science research.

Praising students may be part of being respectful and positive, but praise should be used with caution. It is tempting to praise the quality of a student's contribution, because we expect such praise to make a student feel good and thus more likely to contribute in the future. However, this type of praise can communicate to other students that accuracy is what an instructor values most in students' answers, rather than students articulating their reasoning. It can also communicate that no other answers need to be heard, because such a high-quality answer was already offered (Tanner, 2013). Praise to the whole class, praise that values a contribution (rather than the content of the contribution), and praise that emphasizes hard work over ability can further the goal of encouraging students to contribute (Eddy et al., 2015).

Use Names. Using names to call on students during random call did not meet our threshold for a critical component, but warrants further investigation of its impact on student outcomes in random call. Using students' names is part of instructor immediacy, because it can decrease a sense of distance between students and the instructor (Tanner, 2013). In fact, in a large college biology course in which the instructor endeavored to learn students' names and to call on students using name tents, more students $(78 \%)$ perceived that their names were known than the instructor actually knew (53\%; Cooper et al., 2017). These students reported that it was important to them that the instructor knew their names, because they felt more valued by the instructor and that the instructor cared about them. Students also reported feeling more invested in the course and more comfortable getting help (Cooper et al., 2017). Future work can reveal the role of using names in increasing student comfort with random call.

Ask Questions That Require Higher-Order Cognitive Skills. This component did not meet our threshold for a critical component, but is worth further consideration when we view random call as part of a broader approach to teaching. As some participants indicated, using random call that involves students discussing a question with their peers requires class time that cannot then be spent on other learning activities. Thus, we can expect instructors to want the benefit to student learning to outweigh the cost of the class time used. 
The ICAP framework considers the cognitive demands placed on students by different kinds of questions and the learning gains that can be expected to result from students engaging in these questions (Chi and Wylie, 2014). ICAP refers to interactive, constructive, active, and passive modes of cognitive engagement. Interactive and constructive cognitive engagement involves learners generating knowledge or products that go beyond what has been presented in instructional materials, in collaboration with peers or alone, respectively (Chi and Wylie, 2014). Collectively, interactive and constructive engagement can be referred to as "generative" engagement. Active cognitive engagement involves learners physically manipulating something without adding new knowledge, and passive engagement involves receiving information (Chi and Wylie, 2014). Extensive empirical work indicates that generative engagement leads to greater learning gains than active and passive engagement (Chi, 2009; Menekse et al., 2013). Generative cognitive engagement supports student learning, because it makes knowledge and schema more complete, more coherent, and better linked with other schemas, which facilitates transfer (Chi and Wylie 2014). Thus, a random call question that asks students to engage in generative work is likely a more productive use of class time than questions focused on recall and activity.

Use Random Call Early in the Term and Use Random Call Consistently throughout the Term. These components of random call were used by almost all of our participants, but did not meet the criterion of strong evidence of importance for more than half of users (Table 3). This led us to wonder whether the importance of these components to the success of random call had become tacit (i.e., implicit) knowledge for participants. Using random call early and often may have become a part of their practices to which they no longer give much thought. If that is the case, participants would be less likely to discuss the importance of these components during an interview. The participants who indicated that these practices were important made compelling arguments about how using random call early and often helped students anticipate what would be expected of them, and being able to anticipate random call may make it less anxiety inducing. Furthermore, the benefits of random call, such as holding students accountable, may depend on its regular use. Thus, we propose that future work also consider using random call early and often as potentially critical to the success of implementing random call.

\section{Implications for Instruction}

As we have discussed, additional research will be key to drawing conclusions about the impact of random call on students. Nonetheless, we see a few immediate instructional implications of this research. First, this work reminded us of the importance of carefully considering our rationales for particular instructional practices. We observed that instructors made different choices about how to implement random call based on their rationales for using the practice. Whether a random selection is made with or without replacement is one example of this. Some participants felt that making selections without replacement undermined the goal of holding students accountable for being ready to contribute verbally at any time. Another participant opted to make random selections without replacement, because she wanted students to know they were off the hook after they had been selected. This participant saw diversity of voices as the primary benefit of random call and viewed student anxiety as a serious cost. Thus, it was not as important to her to maintain a constant sense of accountability. Developing a clear rationale for an instructional practice sets up an instructor to make decisions aligned with that rationale and also prepares the instructor to articulate the underlying rationale to students.

Second, we note that our participants were creative in the ways they made random call logistically feasible in their large courses. Based on our participants' success, we assert that random call can be efficiently used in most, if not all, large courses. We are confident that instructors interested in using random call can overcome logistical challenges. One way to tackle anticipated challenges is to learn from an experienced user.

Our third instructional implication is that even a seemingly simple practice like random call relies on detailed teacher knowledge, and instructors can benefit from the expertise of others. Our participants used a carefully chosen set of components in their random call practices and often provided nuanced rationales for their choices. They may have been drawing on expert pedagogical knowledge to inform these choices (Auerbach and Andrews, 2018). Indeed, expert (i.e., effective) active-learning instructors exhibited more knowledge about holding students accountable, creating opportunities for students to generate their own ideas and work, and monitoring and responding to student thinking than did novice (i.e., new) active-learning instructors (Auerbach et al., 2018). We encourage instructors who are new to using random call or who are considering altering their use of random call to learn from the experiences of others. If possible, observing a colleague and asking him or her to explain his or her choices is likely to be a valuable learning experience. This paper provides a window into the thinking of 12 faculty who use random call. We hope that future research will shed additional light on the components of random call that are particularly important for achieving specific outcomes for specific students.

\section{Limitations}

Readers should consider several limitations of this work. First, we have treated decisions that instructors make about random call mostly in isolation of other instructional decisions. This is undoubtedly a misrepresentation of how instructors design their courses, because they must consider how different instructional approaches are woven together to create learning opportunities for students and to create a productive classroom climate. This is akin to studying the dynamics of just one species within an ecosystem of interacting species and abiotic factors. We may have failed to comprehensively understand the thinking that influences participants' decisions regarding random call, because we did not consider other practices they use to achieve the same goals (e.g., accountability, diversity of voices) or other instructional decisions that place limitations on their implementations of random call.

A second limitation is our reliance on self-reporting of instructional practices, rather than observations. There is always a possibility of bias in self-reported data. We remain confident in the trustworthiness of our findings for two reasons. First, we observed two of the participants in a class session that involved random call and were able to confirm that their reports 
of their practices aligned with their observed practices. Second, there is evidence that self-reporting of instructional practices is reliable when participants are asked to report about specific practices rather than more general practices (e.g., Smith et al., 2014). Our interview protocol prompted participants to describe their use of random call in detail, and we used follow-up questions as necessary to clarify details.

Another limitation of our work is that we studied only random call users, many of whom felt strongly about using random call. Our results are not representative of the thinking of the general population of biology instructors or the biology education research community. We recognize that some individuals may feel as passionately about the costs of random call as our participants felt about the benefits of random call. Future studies must investigate student outcomes in different contexts to help us understand the true costs and benefits, and how they apply to different groups of students. We aimed to provide a framework of components of random call that would make it possible for studies of random call to be compared.

Furthermore, we collected data from one former random call user who had experience with the practice but had opted not to use it anymore. These data were not included in this article, but her interview provided useful insights into potential challenges of using random call. Future work would benefit from including more participants who had used random call but have since stopped.

Finally, we only studied instructors who taught courses with 50 or more students. Thus, our results are most relevant in this context. It may be the case that random call achieves different outcomes, is less useful, or has different critical components in courses with fewer students. Students may feel more anonymous to the instructor and to their peers in a larger course, and this may act to decrease or increase the fear of negative evaluation. Studies of the experiences of students will be key to learning how class size interacts with the costs and benefits of random call.

\section{CONCLUSIONS}

Random call has the potential to achieve important goals in undergraduate biology classrooms, including holding students accountable for engaging in work designed to promote their learning and increasing the diversity of student voices heard. Yet this practice may also have negative consequences for some students. Investigating the costs and benefits for different groups of students requires being clear about which components constitute random call and which components are important to which outcomes. The random call components and rationales that emerged from this work are a step in that endeavor. We aim for this work to contribute to Biology Education Research 2.0, in which our community strives to understand what is happening during active learning that makes it work, what "working" means, and for whom (Dolan, 2015).

\section{ACKNOWLEDGMENTS}

Thank you to our research participants for generously offering their time and transparently discussing their teaching. Thanks also to the University of Georgia Biology Education Research Group (BERG) for ongoing feedback and support and to Julie Stanton for raising key questions relevant to this work. Partial support for this work was provided by a grant from the
University of Georgia's Owens Institute for Behavioral Research. Any opinions, findings, and conclusions or recommendations expressed in this paper are those of the authors and do not necessarily reflect the views of the Owens Institute for Behavioral Research. Thank you also to two anonymous peer reviewers whose generous and critical feedback helped us improve this article.

\section{REFERENCES}

Alonzo, A. C., \& Kim, J. (2016). Declarative and dynamic pedagogical content knowledge as elicited through two video-based interview methods. Journal of Research in Science Teaching, 53(8), 1259-1286.

Andrews, T. C., \& Lemons, P. P. (2015). It's personal: Biology instructors prior itize personal evidence over empirical evidence in teaching decisions. CBE-Life Sciences Education, 14(1), ar7.

Andrews, T. C., Auerbach, A. J. J., \& Grant, E. (2019). Exploring the relationship between teacher knowledge and active learning implementation in large college biology courses. CBE-Life Sciences Education, 18(4), ar48. https://doi.org/ 10.1187/cbe.19-01-0010

Andrews, T. M., Leonard, M. J., Colgrove, C. A., \& Kalinowski, S. T. (2011). Active learning not associated with student learning in a random sample of college biology courses. CBE-Life Sciences Education, 10(4), 394405.

Anfara, V. A., Jr,Brown, K. M., \& Mangione, T. L. (2002). Qualitative analysis on stage: Making the research process more public. Educational Researcher, 31(7), 28-38.

Auerbach, A. J., Higgins, M., Brickman, P., \& Andrews, T. C. (2018). Teacher knowledge for active-learning instruction: Expert-novice comparison reveals differences. CBE-Life Sciences Education, 17(1), ar12.

Auerbach, A. J. J., \& Andrews, T. C. (2018). Pedagogical knowledge for active-learning instruction in large undergraduate biology courses: A large-scale qualitative investigation of instructor thinking. International Journal of STEM Education, 5(1), 19

Broeckelman-Post, M., Johnson, A., \& Schwebach, J. R. (2016). Calling on students using notecards: Engagement and countering communication anxiety in large lecture. Journal of College Science Teaching, 45(5), 27

Brooks, B. J., \& Koretsky, M. D. (2011). The influence of group discussion on students' responses and confidence during peer instruction. Journal of Chemical Education, 88(11), 1477-1484.

Cavanagh, A. J., Aragón, O. R., Chen, X., Couch, B. A., Durham, M. F., Bobrownicki, A., ... \& Graham, M. J. (2016). Student buy-in to active learning in a college science course. CBE-Life Sciences Education, 15(4), ar76.

Cavanagh, A. J., Chen, X., Bathgate, M., Frederick, J., Hanauer, D. I., \& Graham, M. J. (2018). Trust, growth mindset, and student commitment to active learning in a college science course. CBE-Life Sciences Education, 17(1), ar10.

Charmaz, K. (2006). Constructing grounded theory: A practical guide through qualitative analysis. Thousand Oaks, CA: Sage.

Chi, M. T. (2009). Active-constructive-interactive: A conceptual framework for differentiating learning activities. Topics in Cognitive Science, 1(1), 73-105.

Chi, M. T., \& Wylie, R. (2014). ICAP: A hypothesis of differentiated learning effectiveness for four modes of engagement activities. Educational Psychologist, 49(4), 219-243.

Cooper, K. M., \& Brownell, S. E. (2016). Coming out in class: Challenges and benefits of active learning in a biology classroom for LGBTQIA students. CBE-Life Sciences Education, 15(3), ar37.

Cooper, K. M., Downing, V. R., \& Brownell, S. E. (2018). The influence of active learning practices on student anxiety in large-enrollment college science classrooms. International Journal of STEM Education, 5(1), 23.

Cooper, K. M., Haney, B., Krieg, A., \& Brownell, S. E. (2017). What's in a name? The importance of students perceiving that an instructor knows their names in a high-enrollment biology classroom. CBE-Life Sciences Education, 16(1), ar8.

Dallimore, E. J., Hertenstein, J. H., \& Platt, M. B. (2004a). Classroom participation and discussion effectiveness: Student-generated strategies. Communication Education, 53(1), 103-115. 
Dallimore, E. J., Hertenstein, J. H., \& Platt, M. B. (2004b). Faculty-generating strategies for "cold calling" use: A comparative analysis with student recommendations. Journal on Excellence in College Teaching, 16(1), 23-62.

Dallimore, E. J., Hertenstein, J. H., \& Platt, M. B. (2013). Impact of cold-calling on student voluntary participation. Journal of Management Education, 37(3), 305-341.

Dancy, M., Henderson, C., \& Turpen, C. (2016). How faculty learn about and implement research-based instructional strategies: The case of peer instruction. Physical Review Physics Education Research, 12(1), 010110.

Dolan, E. L. (2015). Biology Education Research 2.0. CBE-Life Sciences Education, 14(4), ed1.

Eddy, S. L., Brownell, S. E., Thummaphan, P., Lan, M. C., \& Wenderoth, M. P. (2015). Caution, student experience may vary: Social identities impact a student's experience in peer discussions. CBE-Life Sciences Education, 14(4), ar45.

Eddy, S. L., Brownell, S. E., \& Wenderoth, M. P. (2014). Gender gaps in achievement and participation in multiple introductory biology classrooms. CBE-Life Sciences Education, 13(3), 478-492.

Eddy, S. L., \& Hogan, K. A. (2014). Getting under the hood: How and for whom does increasing course structure work? CBE-Life Sciences Education, 13(3), 453-468.

England, B. J., Brigati, J. R., \& Schussler, E. E. (2017). Student anxiety in introductory biology classrooms: Perceptions about active learning and persistence in the major. PLOS ONE, 12(8), e0182506.

England, B. J., Brigati, J. R., Schussler, E. E., \& Chen, M. M. (2019). Student anxiety and perception of difficulty impact performance and persistence in introductory biology courses. CBE-Life Sciences Education, 18(2), $\operatorname{ar} 21$

Estrada, M., Eroy-Reveles, A., \& Matsui, J. (2018). The influence of affirming kindness and community on broadening participation in STEM career pathways. Social Issues and Policy Review, 12(1), 258-297.

Estrada, M., Young, G. R., Nagy, J., Goldstein, E. J., Ben-Zeev, A., Márquez Magaña, L., \& Eroy-Reveles, A. (2019). The influence of microaffirmations on undergraduate persistence in science career pathways. CBE-Life Sciences Education, 18(3), ar40.

Grunspan, D. Z., Eddy, S. L., Brownell, S. E., Wiggins, B. L., Crowe, A. J., \& Goodreau, S. M. (2016). Males under-estimate academic performance o their female peers in undergraduate biology classrooms. PLOS ONE, 11(2), e0148405

Hall, G. E., \& Loucks, S. F. (1978). Innovation configurations: Analyzing the adaptations of innovations. Research and Development Center for Teacher Education, University of Texas, Austin, Texas.

Heimberg, R. G., Brozovich, F. A., \& Rapee, R. M. (2010). A cognitive behavioral model of social anxiety disorder update and extension. In Hofmann, S G., \& DiBartolo, P. M. (Eds.), Social anxiety: Clinical, developmental, and social perspectives (2nd ed., pp. 395-422). Amsterdam: Elsevier.

Keeley, J., Zayac, R., \& Correia, C. (2008). Curvilinear relationships between statistics anxiety and performance among undergraduate students: Evidence for optimal anxiety. Statistics Education Research Journal, 7(1), $4-15$

Knight, J. K., Wise, S. B., \& Sieke, S. (2016). Group random call can positively affect student in-class clicker discussions. CBE-Life Sciences Education, 15(4), ar56
Knight, J. K., Wise, S. B., \& Southard, K. M. (2013). Understanding clicker discussions: Student reasoning and the impact of instructional cues. CBELife Sciences Education, 12(4), 645-654.

Lohr, S. L. (1999). Sampling: Design and analysis. Pacific Grove, CA: Brooks.

Martin, S. N., Milne, C., \& Scantlebury, K. (2006). Eye-rollers, risk-takers, and turn sharks: Target students in a professional science education program. Journal of Research in Science Teaching, 43, 819-851.

Mays, N., \& Pope, C. (2000). Assessing quality in qualitative research. BMJ, $320(7226), 50-52$

Mazur, E., \& Hilborn, R. C. (1997). Peer instruction: A user's manual (Vol. 5). Upper Saddle River, NJ: Prentice Hall.

McCall, L. (2008). The complexity of intersectionality. In Grabham, E., Cooper, D., Krishnadas, J., \& Herman, D. (Eds.), Intersectionality and beyond (pp. 65-92). New York, NY: Routledge-Cavendish.

McClain, S., \& Cokley, K. (2017). Academic disidentification in Black college students: The role of teacher trust and gender. Cultural Diversity and Ethnic Minority Psychology, 23(1), 125.

Mehrabian, A. (1971). Silent messages (Vol. 8). Belmont, CA: Wadsworth.

Menekse, M., Stump, G. S., Krause, S., \& Chi, M. T. (2013). Differentiated overt learning activities for effective instruction in engineering classrooms. Journal of Engineering Education, 102(3), 346-374.

Saldaña, J. (2009). The coding manual for qualitative researchers. Thousand Oaks, CA: Sage.

Scanlon, E., Roman, B. Z., Ibadlit, E., \& Chini, J. J. (2019). A method for analyzing instructors' purposeful modifications to research-based instructional strategies. International Journal of STEM Education, 6(1), 12.

Seidel, S. B., Reggi, A. L., Schinske, J. N., Burrus, L. W., \& Tanner, K. D. (2015) Beyond the biology: A systematic investigation of noncontent instructor talk in an introductory biology course. CBE-Life Sciences Education 14(4), ar43.

Seidel, S. B., \& Tanner, K. D. (2013). "What if students revolt?"-considering student resistance: Origins, options, and opportunities for investigation. CBE-Life Sciences Education, 12(4), 586-595.

Shenton, A. K. (2004). Strategies for ensuring trustworthiness in qualitative research projects. Education for Information, 22(2), 63-75.

Smith, M. K., Vinson, E. L., Smith, J. A., Lewin, J. D., \& Stetzer, M. R. (2014). A campus-wide study of STEM courses: New perspectives on teaching practices and perceptions. CBE-Life Sciences Education, 13(4), 624-635.

Smith, M. K., Wood, W. B., Adams, W. K., Wieman, C., Knight, J. K., Guild, N., \& $\mathrm{Su}$, T. T. (2009). Why peer discussion improves student performance on in-class concept questions. Science, 323(5910), 122-124

Stains, M., \& Vickrey, T. (2017). Fidelity of implementation: An overlooked ye critical construct to establish effectiveness of evidence-based instructional practices. CBE-Life Sciences Education, 16(1), rm1.

Tanner, K. D. (2013). Structure matters: Twenty-one teaching strategies to promote student engagement and cultivate classroom equity. CBE-Life Sciences Education, 12(3), 322-331.

Vickrey, T., Rosploch, K., Rahmanian, R., Pilarz, M., \& Stains, M. (2015). based implementation of peer instruction: A literature review. CBE-Life Sciences Education, 14(1), es3.

Yerkes, R. M., \& Dodson, J. D. (1908). The relation of strength of stimulus to rapidity of habit-formation. Journal of Comparative Neurology and Psychology, 18(5), 459-482. 